\title{
Efficacy of letrozole versus clomiphene citrate for infertile women with polycystic ovary syndrome
}

\author{
Sinan S. Ay ${ }^{1}$, Ozer Birge ${ }^{1 *}$, Mehmet S. Bakir ${ }^{1}$, Ayse E. Yumru²
}

\author{
${ }^{1}$ Department of Obstetrics and Gynecology, Akdeniz University, Antalya, Turkey \\ ${ }^{2}$ Department of Obstetrics and Gynecology, Şişli Hamidiye Etfal Training and Research Hospital, İstanbul, Turkey
}

Received: 30 May 2021

Accepted: 03 July 2021

\section{*Correspondence:}

Dr. Ozer Birge,

E-mail: ozbirge@gmail.com

Copyright: ( ) the author(s), publisher and licensee Medip Academy. This is an open-access article distributed under the terms of the Creative Commons Attribution Non-Commercial License, which permits unrestricted non-commercial use, distribution, and reproduction in any medium, provided the original work is properly cited.

\begin{abstract}
Background: The aim was to compare ovulation induction protocols in anovulatory patients, who make up a significant percentage of infertility patients, and to determine the most appropriate treatment for patients in the clinic based on the findings.

Methods: The effectiveness of clomiphene citrate (CC) and letrozole (aromatase inhibitor) in ovulation induction treatments were retrospectively compared in patients who applied for infertility in the last 5 years and were found to be anovulatory. 20 of these patients were being treated with clomiphene citrate, while the 18 were being treated with letrozole.

Results: The study included a total of 38 anovulatory infertile patients. The mean age of the patients was found to be 29.3. When the endometrial thicknesses (ET) after the treatment were compared, the first group's mean EC was 6.1, while that of the second group was 9.05. The endometrial thicknesses measured after the treatments were found to be significantly different, which were consistent with other studies in the literature. Post-treatment ovulation responses were similar with $55 \%$ in both groups. In the evaluation of pregnancy outcomes, $20 \%$ of pregnancy was achieved in the first group and $33 \%$ in the second group.

Conclusions: The use of letrozole, an aromatase inhibitor, may be suggested as an alternative to CC in the ovulation induction protocol in our clinical practice, particularly in obese patients.
\end{abstract}

Keywords: Infertility, Polycystic ovary syndrome, Anovulation, Letrozole, Clomiphene citrate

\section{INTRODUCTION}

Polycystic ovary syndrome (PCOS) is defined as the presence of oligo-anovulation and/or hyperandrogenism proven by clinical or laboratory findings and/or polycystic ovary image on ultrasonography. It affects $5-10 \%$ of women in the reproductive period and is the most common cause of anovulatory infertility. ${ }^{1}$ Although it is a complex metabolic disorder, ovulation induction is most commonly used when the hypothalamic-pituitary axis is affected. Clomiphene citrate (CC), a selective oestrogen receptor blocker, is the most widely used agent for this purpose. CC causes ovulation induction by blocking oestrogen receptors in the hypothalamus, increasing endogenous gonadotropin secretion with a negative feedback effect. $\mathrm{CC}$ has received some criticism (reservations) despite its use as a first-line treatment for anovulatory infertile patients. For instance, live pregnancy rates of around $20 \%$ and multiple pregnancy rates of 3-8\% can be considered as mood disorders and hot flashes. ${ }^{2} \mathrm{CC}$ has been heavily criticized in terms of cost efficiency due to the high rates of multiple pregnancies as compared to natural cycles. ${ }^{3}$

An effective, easy and safe ovulation induction is one of the most important public health problems. ${ }^{4}$ Therefore, alternative treatment modalities to $\mathrm{CC}$ have been 
investigated. Metformin, for example, has been used in anovulatory infertile patients, but its superiority to $\mathrm{CC}$ has yet to be shown. ${ }^{2,5}$ Aromatase inhibitors (AI) are one of the selective oestrogen receptor blockers that have been used for this purpose in recent years. ${ }^{6}$ The most important advantage of AI arise from the fact that they have a positive effect on the endometrium, have fewer multiple pregnancy rates, have less half-life compared to $\mathrm{CC}$, are easier to clear from the body, and mimic physiological ovulatory hyperstimulation because they have fewer and tolerable side effects. ${ }^{6}$ However, teratogenic side effects made AI controversial. Concerns caused by their teratogenic effects have been mitigated by two large multicentric studies and have been shown to be reliable. ${ }^{7,8}$ They have been shown to be a serious alternative to $\mathrm{CC}$ in terms of both live pregnancy and ovulation rates in a randomized controlled trial (RCT) compared to CC because of the potential advantages of their AIs. ${ }^{9}$ Again, in another current RCT, pregnancy rates were shown to be approximately 2 times higher in the letrozole group compared to the CC group (42\% versus $20 \%$, respectively), and mono-follicular development was shown to be better in the letrozole group. ${ }^{10}$

This study aims to compare the ovulation induction treatments performed with $\mathrm{CC}$, which is a common induction method in anovulatory patients, and letrozole, an aromatase inhibitor, to retrospectively evaluate the treatment results of our clinic and our patients, and to obtain information to improve the follow-up and treatment of such patients as a result of our findings. These low-cost protocols, which are not difficult to apply and follow, rely on the patients' cooperation and improve their motivation to become pregnant with the follow-up and treatment they are receiving.

\section{METHODS}

In this retrospective study, $\mathrm{CC}$ and letrozole (AI) and ovulation induction treatments were compared in PCOS patients who applied to Istanbul Şişli Hamidiye Etfal training and research hospital due to infertility within 5 years covering the years January 2014 to January 2018 and were found to be anovulatory. Ethical approval was obtained from the İstanbul Şişli Hamidiye Etfal training and research ethics committee with reference number 2013-ISH-20 and date KAEK-110/10.02.2013. Detailed anamneses of the patients included in the study group were taken and detailed physical examinations were performed. Our patients have never been treated for infertility before and are now receiving care for the first time.

\section{Inclusion criteria}

Patients with primary infertile anovulatory PCOS were included in the study. For the diagnosis of patients with PCOS, besides oligo-anovulation, the presence of hyperandrogenism findings (clinical or laboratory) and/or polycystic ovaries (presence of 12 antral follicles under 10 $\mathrm{mm}$ or at least one ovary volume of $10 \mathrm{~cm}^{3}$ ) in ultrasonography, which are among the modified Rotterdam criteria, were used. ${ }^{1}$ While basal follicle stimulating hormone $(\mathrm{FSH})<13$, thyroid stimulating hormone (TSH) and prolactin values within normal limits, not having sexual dysfunction, having healthy sexual activities were the inclusion criteria, the exclusion criteria from the study were the presence of clinically significant systemic or endocrine disease, the presence of infertility due to the malefactor, previous ovulation induction, controlled ovarian hyperstimulation $(\mathrm{COH})$ or assisted reproductive techniques (ART) application, those with anatomical uterine, tubal, endometrial, peritoneal abnormalities, and the presence of an infectious pattern with chlamydia antibody positivity.

All of the patients in the sample were extensively examined to ensure that there were no other factors of infertility than anovulation. Spermiogram examinations obtained from spouses in terms of male factor were performed, and the results were observed to be normal. To rule out anatomical anomalies, ultrasonography and hysterosalpingography were performed, and it was concluded that there were no tubal uterine peritoneal risk factors. To assess if the ovarian reserves were adequate, antral follicle counts (AFC), AMH tests, serum FSH, and estradiol (E2) tests were performed.

At the initial examination of the selected patients, their identity and age were determined, and their obstetric and gynaecological histories and menstrual cycle patterns were questioned. In physical examinations, blood pressure, height, weight, and body mass index (BMI) (weight/height ${ }^{2}$ ) were measured. Secondary sex character development was evaluated. Routine pelvic examinations were performed. The following laboratory values were checked prior to treatment. Fasting blood glucose, urea, serum glutamic oxaloacetic transaminase (SGOT), serum glutamic pyruvic transaminase (SGPT) (after 12 hours of fasting, $10 \mathrm{ml}$ of venous blood was sampled), and thyroid function tests were performed. In the early follicular phase (2-3 days), basal ultrasonography (USG) was performed and uterine dimensions, endometrial thickness, ovarian dimensions, follicle number, and diameters were measured. For this process, B-K Medical (type 2101 B-K Medical A/S Gentofte Denmark) brand ultrasonography device and a $5 \mathrm{MHz}$ vaginal probe were used. Basal (3rd day) FSH, leutinizing hormone ( $\mathrm{LH})$, E2, prolactin values were measured.

Each patient's ovaries, tubal structures, and endometrial structures were all evaluated using basal ultrasonography prior to treatment. Ultrasonography follow-up was done every 2-3 days during the patients' treatment and ovulation follow-up. Following the detection of mature follicle development reaching 18-20 mm diameter, follicle rupture was induced with pregnyl (b-hCG) 10000 IU. They were invited to a serial ultrasonography and examination follow-up every two days to see if ovulation had occurred. All patients were reached at the appropriate time at the follow-up process. 
Patients undergoing $\mathrm{COH}$ were carefully followed in terms of ovarian hyperstimulation syndrome (OHSS). On the 14th and 16th days of ovulation, the b-hCG test was used to determine whether pregnancy occurred or not. In pregnant women, mid-luteal (21st day) progesterone levels were measured, and luteal phase support progesterone 200 $\mathrm{mg} /$ day oral treatment was provided. Preconceptional 400 $\mathrm{mg} /$ day oral folic acid was provided to all patients because they were trying to get pregnant.

Treatment protocols were made in accordance with American college of obstetricians and gynecologists (ACOG) 2002 bulletin, treatment in ovulation induction protocols. ${ }^{11}$

\section{Clomiphene citrate treatment protocol}

It is started on the 3rd-5th day of menstruation and 50 $\mathrm{mg} /$ day for 5 days. If ovulation is not observed, the dose is increased after the second cycle (50 $\mathrm{mg}$ to $100 \mathrm{mg}$ ). The maximum recommended dose is $150 \mathrm{mg} / \mathrm{day} .{ }^{11} \mathrm{~A}$ maximum of 6 cycles is applied. Starting 5 days after the last dose, regular coitus is recommended for 1 week. When the follicle diameter is $18-20 \mathrm{~mm}$ in USG follow-up, $10000 \mathrm{IU}$ hCG is applied.

\section{Letrozole treatment protocol}

It is started on the 2nd-5th day of menstruation as 2.5 - 5 $\mathrm{mg} /$ day for 5 days. The optimal dose is $5 \mathrm{mg} /$ day. A maximum of 6 cycles is applied. When the follicle diameter is $18-20 \mathrm{~mm}$ in USG follow-up, $10000 \mathrm{IU}$ hCG is applied. Group 1 included 20 patients induced by CC, and group 2 included 18 patients induced by letrozole.

\section{Statistical analysis}

The analysis of the data was performed using the statistical package for the social sciences (SPSS) package program 20.0 version. The descriptive data were presented using frequency, percentage, mean, and standard deviation, median, minimum, and maximum values.

The compliance of the variables to normal distribution was examined using the Shapiro-Wilk test based on the number of patients in the groups. When the sample size and compliance tests with normal distribution were examined, nonparametric tests were preferred as analysis methods. For the comparison of age and continuous variables between groups, the Mann-Whitney $U$ test was used. Continuity corrected Chi-square test and Fisher's exact test were used to compare categorical variables among treatment groups. The cases where the $p$ value is below 0.05 were considered statistically significant.

\section{RESULTS}

$\mathrm{COH}$ with letrozole was performed in 18 of the study's patients, while $\mathrm{COH}$ with $\mathrm{CC}$ was performed in 20. The median age of the letrozole group was 29 (min: 25, max: 36 ), and that of the CC group was the same (min: 24, max: 36 ), so there was no statistically significant difference between them $(\mathrm{p}=0.607)$. The clinical and demographic characteristic risk factors of the patients are given in Table 1. The median infertile time of both groups was 3 years and there was no difference between them $(p=0.354)$. While the rate of smokers in the Letrozole group was $27.8 \%$, it was $35 \%$ in the CC group, and there was no statistically significant difference between them either $(p=0.734)$. When both groups were compared in terms of increase in endometrial thickness, the median endometrial thickness was found to be 9 (min: 7 , max: 12) in the letrozole group, while it was 6 (min: 4 , max: 8 ) in the CC group, and there was a statistically significant difference $(p=0.001)$. When the BMI of the two groups was compared, the letrozole group was observed to have a statistically higher BMI ( $\mathrm{p}=0.012)$ (Table 1).

When the treatment groups were evaluated in terms of the ovulation response, it was found that 10 patients in the letrozole group and 11 patients in the $\mathrm{CC}$ group had ovulation responses, but the difference was not statistically significant $(\mathrm{p}=0.615)$. Table 2 shows a detailed comparison of the treatment outcomes of both groups. While the number of pregnancies observed in the letrozole group was $6(33.3 \%)$, it was $4(20 \%)$ in the CC group and there was no statistically significant difference $(\mathrm{p}=0.573)$. When the live pregnancy results were examined, it was observed that $4(66.7 \%)$ live pregnancies occurred in the letrozole group and $3(75 \%)$ live pregnancies in the CC group, and there was no statistically significant difference between the two groups, as well $(\mathrm{p}=0.778)$ (Table 2).

Table 1: Clinical and demographic characteristic risk factors of the patients.

\begin{tabular}{|llll|}
\hline Characteristic & $\begin{array}{l}\text { Letrozole group, group: 1, } \\
\mathbf{n = 1 8}\end{array}$ & $\begin{array}{l}\text { CC group, group: 2, } \\
\mathbf{n = 2 0}\end{array}$ & P value \\
\hline Age (year) (median) & $29(25-36)$ & $29(24-33)$ & 0.607 \\
\hline Duration of infertility (years) (median) & $3(2-4)$ & $3(2-5)$ & 0.354 \\
\hline Endometrial thickness & $9(7-12)$ & $6(4-8)$ & 0.001 \\
\hline BMI & $28.5(23-31)$ & $26(23-30)$ & 0.012 \\
\hline Smoking status & & & 0.734 \\
\hline Yes & $5(27.8 \%)$ & $7(35.0 \%)$ & $13(65.0 \%)$ \\
\hline No & $13(72.2 \%)$ & & \\
\hline
\end{tabular}


Table 2: Comparison of both patient groups in terms of treatment results.

\begin{tabular}{|c|c|c|c|}
\hline Parameter & Group $1(\%)$ & Group $2(\%)$ & $P$ value \\
\hline \multicolumn{4}{|c|}{ Ovulation response } \\
\hline Yes & $10(55.6)$ & $11(55.0)$ & \multirow{2}{*}{0.615} \\
\hline No & $8(44.4)$ & $9(45.0)$ & \\
\hline \multicolumn{4}{|c|}{ Pregnancy status } \\
\hline Yes & $6(33.3)$ & $4(20.0)$ & \multirow{2}{*}{0.573} \\
\hline No & $12(66.7)$ & $16(80.0)$ & \\
\hline \multicolumn{4}{|c|}{ Ongoing pregnancy (live birth) } \\
\hline Yes & $4(66.7)$ & $3(75.0)$ & \multirow{2}{*}{0.778} \\
\hline No & $2(33.3)$ & $1(25.0)$ & \\
\hline
\end{tabular}

\section{DISCUSSION}

Polycystic ovary syndrome is the most common cause of anovulatory infertility, with a prevalence of $6-21 \%$ in the general population and a diagnosis rate of $70 \%$, particularly in anovulatory infertile cases. ${ }^{12-14}$ Infertility is a condition seen in $10-15 \%$ of couples and requires treatment. In this context, in this study, we compared clomiphene citrate and Letrozole, an aromatase inhibitor, in patients with polycystic ovarian anovulation.

Considering the general results of our study, when the use of letrozole as ovulation induction was compared with CC, a statistically significant difference was found in the increase in endometrial thickness in overweight obese patients $(\mathrm{p}=0.001)$, and it was observed that ovulation rates and pregnancy rates were higher. However, there was no statistically significant difference detected between these rates.

Our study group consists of patients between the ages of 25-35. The mean age of the first group was found to be 29, and that of the second group was 29.6. The age distribution of the patients in both groups is similar. One of the most important parameters in infertility is age. It is known that the ovarian reserve and therefore the fertility of the patients progressively decrease with age. ${ }^{15,16}$

While toxic agents such as nicotine are thought to have a negative impact on infertility, there is yet to be a definitive study. Smoking patients made up $35 \%$ of the first group and $27 \%$ of the second group in our study. The number of smokers in the first group is $29 \%$ higher than in the second.

Bodyweight is closely related to the ovulation mechanism of women. Peripheral adipose tissue and central obesity disrupt the hormonal cycle and associated hyperinsulinemia negatively affects the menstrual arc. When anovulatory patients with obesity and PCOS return to acceptable body weights after diet and exercise, about one-third of them spontaneously recover normal menstrual cycles. For this reason, obese patients, who make up the majority of anovulatory patients, are advised to lose weight through a combination of lifestyle changes, exercise, and diet, as well as scheduled follow-up and treatment. ${ }^{17}$
Sharief et al. concluded in their study on 57 obese patients that letrozole should be preferred primarily in obese patients who will undergo ovulation induction due to its endometrial advantage. ${ }^{18}$ When our study was evaluated in terms of BMI, no significant difference was found.

In a controlled randomized prospective study of 268 anovulatory patients, Liu et al compared letrozole and CC and found that the two groups had similar ovulation responses. ${ }^{19}$ In their ovulation induction study on 57 anovulatory patients, Wang et al discovered that the letrozole group had a higher live birth rate. ${ }^{20}$ When $\mathrm{CC}$ and Aromatase inhibitors were compared in similar studies, the results showed that ovulation responses were similar. In our study, both groups had a $55 \%$ ovulation rate in terms of ovulation responses, indicating that ovulation results were identical.

Ovulation was reported to be $60 \%$ in the letrozole group and $32 \%$ in the CC group in another study $(\mathrm{p}=0.009) .^{21}$ The ovulation rate in our study was $55.6 \%$ in the letrozole group and $55 \%$ in the $\mathrm{CC}$ group, which was consistent with all these studies, but the difference between the two groups was not statistically significant $(p=0.615)$. All of these studies were comparable to ours, and it can be concluded that letrozole has higher ovulation rates for PCOS.

Endometrial thickness is a valuable criterion for successful implantation and has a direct impact on fertility. The endometrial thickness after ovulation should be greater than $8 \mathrm{~mm}$. Previous studies have shown that patients who received this treatment had thin endometrial thickness due to its antiestrogenic effects on the $\mathrm{CC}$ endometrium. The results obtained in our study also support these findings. While the mean endometrial thickness of the first group patients was $6.1 \mathrm{~mm}$, it was found to be $9.05 \mathrm{~mm}$ in the second group. Because of the known antiestrogenic effect of $\mathrm{CC}$ on the endometrium, cervix, and vagina, the endometrial thickness of these patients was significantly thinner $(32 \%)$ compared to the patients who received letrozole treatment.

In a study published in 2020, a total of 220 cases with PCOS were divided into two groups, and after 12 months of follow-up with letrozole and $\mathrm{CC}$ treatment cycles, pregnancy was detected in $45(40.9 \%)$ patients using 
letrozole and 27 (24.5\%) patients using CC were pregnant. This difference between the two groups was found to be statistically significant $(\mathrm{p}=0.007)$. When evaluated in terms of endometrial thickness, it was found to be $8.3 \pm 2.6$ in the CC group and $11.8 \pm 2.7$ in the letrozole group, and the difference between the two groups was considered statistically significant $(\mathrm{p}=0.001) .{ }^{22}$

Pregnancy rates with letrozole ranged from $16-27.3 \%$ in several studies of similar size and type to ours, while pregnancy rates in the CC group ranged from 13-20.8\%. Although pregnancy rates were high in the letrozole group, this increase was not statistically significant. ${ }^{23-25}$

The findings of some studies on pregnancy rates were similar, while others found that inductions with aromatase inhibitors resulted in higher rates. In our study, these rates were determined as $20 \%$ for the first group and $33 \%$ for the second group, and although the rates seemed numerically higher in the second group, it was concluded that they were similar as a result of statistical analysis.

When the live birth rates of the two groups were compared, abortion was observed in $1(25 \%)$ of 4 pregnancies in the first group and $2(33 \%)$ of 6 pregnancies in the second. When the data were compared statistically, the abortion rates and live birth rates of both groups were found to be similar. In terms of live birth and abortion, it is argued that the abortion rates may have increased due to the antiestrogenic effect of CC on the endometrium. In the study conducted by Hashim et al similar pregnancy rates were found, and it was determined that larger groups were needed. ${ }^{26}$

Letrozole and CC were compared as ovulation induction in a recent study that used a treatment procedure similar to ours but on a greater number of cases, and significantly improved endometrial thickness, higher ovulation, and clinical and live pregnancy rates were revealed. ${ }^{27}$ As compared to the data from our study, the increase in endometrial thickness was significantly different despite the ovulation and pregnancy rates were higher. However, we believe that if the number of cases is increased, the same findings will be obtained.

While the retrospective nature of our study, the small number of our cases, and the insufficient follow-up of the beginning of the treatment and the process after the detection of pregnancy indicate its limitations, the facts that all cases included in our study were diagnosed with the same criteria, the demographic and laboratory results were similar, and the treatment protocol applied was at international standards and similar indicates the strength of our study.

\section{CONCLUSION}

Higher ovulation rates, improved endometrial thickness, and higher pregnancy rates were found to be significantly higher in letrozole, an aromatase inhibitor, as an alternative to $\mathrm{CC}$, which is widely used in the ovulation induction protocol, and it can be recommended for use in clinical practice in the ovulation induction protocol.

Funding: No funding sources

Conflict of interest: None declared

Ethical approval: The study was approved by the Institutional Ethics Committee

\section{REFERENCES}

1. Rotterdam ESHRE/ASRM-Sponsored PCOS Consensus Workshop Group. Revised 2003 consensus on diagnostic criteria and long-term health risks related to polycystic ovary syndrome. Fertil Steril. 2004;81:19-25.

2. Legro RS, Barnhart HX, Schlaff WD. Clomiphene, metformin, or both for infertility in the polycystic ovary syndrome. N Engl J Med. 2007;356:551-66.

3. Kamphuis EI, Bhattacharya S, van der Veen F, Mol BW, Templeton A. Are we overusing IVF? BMJ. 2014;348:252.

4. Thessaloniki ESHRE/ASRM-Sponsored PCOS Consensus Workshop Group. Consensus on infertility treatment related to polycystic ovary syndrome. Fertil Steril. 2008;89:505-22.

5. Tang T, Lord JM, Norman RJ, Yasmin E, Balen AH. Insulin-sensitising drugs (metformin, rosiglitazone, pioglitazone, D-chiro-inositol) for women with polycystic ovary syndrome, oligo amenorrhoea, and subfertility. Cochrane Database Syst Rev. 2012;5:CD003053.

6. Casper RF, Mitwally MF. Review: aromatase inhibitors for ovulation induction. J Clin Endocrinol Metab. 2006;91:760-71.

7. Tulandi T, Martin J, Al-Fadhli R, Kabli N, Forman R, Hitkari J, et al. Congenital malformations among 911 newborns conceived after infertility treatment with letrozole or clomiphene citrate. Fertil Steril. 2006;85(6):1761-5.

8. Forman R, Gill S, Moretti M, Tulandi T, Koren G, Casper R. Fetal Safety of Letrozole and Clomiphene Citrate for Ovulation Induction. J Obstet Gynaecol Can. 2007;29(8):668-71.

9. Legro RS, Brzyski RG, Diamond MP. Letrozole versus clomiphene for infertility in the polycystic ovary syndrome. N Engl J Med. 2014;371(2):119-29.

10. Bansal S, Goyal M, Sharma C, Shekhar S. Letrozole versus clomiphene citrate for ovulation induction in anovulatory women with polycystic ovarian syndrome: A randomized controlled trial. Int $\mathbf{J}$ Gynaecol Obstet. 2021;152(3):345-50.

11. ACOG Committee on Practice Bulletins-Gynecology. ACOG Practice Bulletin. Clinical management guidelines for obstetrician-gynecologists number 34, February 2002. Management of infertility caused by ovulatory dysfunction. American College of Obstetricians and Gynecologists. Obstet Gynecol. 2002;99(2):347-58. 
12. Joham AE, Teede HJ, Ranasinha S, Zoungas S, Boyle J. Prevalence of infertility and use of fertility treatment in women with polycystic ovary syndrome: data from a large community-based cohort study. J Women's Health (Larchmt). 2015;24(4):299-307.

13. Roy KK, Baruah J, Singla S, Sharma JB, Singh N, Jain SK, et al. A prospective randomized trial comparing the efficacy of Letrozole and Clomiphene citrate in induction of ovulation in polycystic ovarian syndrome. J Hum Reprod Sci. 2012;5(1):20-5.

14. Dewailly D. Diagnostic criteria for PCOS: Is there a need for a rethink? Best Pract Res Clin Obstet Gynaecol. 2016;37:5-11.

15. Kiddy DS, Hamilton-Fairley D, Bush A, Short F, Anyaoku V, Reed MJ, Franks S. Improvement in endocrine and ovarian function during dietary treatment of obese women with polycystic ovary syndrome. Clin Endocrinol. 1992;36:105-11.

16. Dickey RP, Taylor SN, Curole DN, Rye PH, Lu PY, Pyrzak R. Relationship of clomiphene dose and patient weight to successful treatment. Hum Reprod. 1997;12:449-53.

17. Sturrock ND, Lannon B, Fay TN. Metformin does not enhance ovulation induction in clomiphene-resistant polycystic ovary syndrome in clinical practice. $\mathrm{Br} \mathrm{J}$ Clin Pharmacol. 2002;53:469-73.

18. Sharief M, Nafee NR, Comparison of letrozole and clomiphene citrate in women with polycystic ovaries undergoing ovarian stimulation, J Pak Med Assoc. 2015;65(11):1149-52.

19. Fleming R, Hopkinson ZE, Wallace AM, Greer IA, Sattar N. Ovarian function and metabolic factors in women with oligomenorrhea treated with metformin in a randomized double blind placebo-controlled trial. J Clin Endocrinol Metab. 2002;87:569-74.

20. Liu C, Feng G, Huang W, Wang Q, Yang S, Tan J, Fu J, Liu D. Gynecol Endocrinol. 2017:1-5.

21. Dehbashi S, Kazerooni T, Robati M, Alborzi S, Parsanezhad ME, et al. Comparison of the effects of letrozole and clomiphene citrate on ovulation and pregnancy rate in patients with polycystic ovary syndrome. Iranian J Med Sci. 2009;34:23-8.

22. Najafi PZ, Noghabi SP, Afzali N, Mohammadzadeh S. Comparing the effect of clomiphene citrate and letrozole on ovulation induction in infertile women with polycystic ovary syndrome. J Pak Med Assoc. 2020;70(2):268-71.

23. Zadehmodares S, Niyakan M, Sharafy SA, Yazdi MH, Jahed F. Comparison of treatment outcomes of infertile women by clomiphene citrate and letrozole with gonadotropins underwent intrauterine insemination. Acta Med Iran. 2012;50(1):18-20.

24. Nanbakhsh F, Hagishafieha M, Tabatabe E, Mazloomi P, Oshnouei S. A Comparison Of Effects Of Admınısterıng Letrezol And Chlomıphen Citrate On Infertility Treatment Outcomes In Patients With Polysıstıc Ovarıan Syndrome. J Urmia Univer Med Sci. 2013;24:543-9.

25. El Bigawy AF, Fouda UM, Wahab HA. A randomized trial of letrozole versus clomiphene citrate in induction of ovulation in patients with polycystic ovary syndrome (PCOS). Middle East fertility society J. 2008;13:52-6.

26. Abu Hashim H. Aromatase Inhibitors for Endometriosis-Associated Infertility; Do We Have Sufficient Evidence? İnt J Fertil Steril. 2016;10(3):270-7.

27. Sakar MN, Oglak SC. Letrozole is superior to clomiphene citrate in ovulation induction in patients with polycystic ovary syndrome. Pak J Med Sci. 2020;36(7):1460-5.

Cite this article as: Ay SS, Birge O, Bakir MS, Yumru AE. Efficacy of letrozole versus clomiphene citrate for infertile women with polycystic ovary syndrome. Int J Reprod Contracept Obstet Gynecol 2021;10:2944-9. 